\title{
Data Acquisition and Image Processing Related to Changes in Nuclear Cardiology Devices
}

\author{
Yasuyuki Takahashi, $\mathrm{PhD}$
}

Received: April 19, 2015/Revised manuscript received: June 18, 2015/Accepted: June 19, 2015

(C) The Japanese Society of Nuclear Cardiology 2015

\section{Abstract}

Forty years have passed since the introduction of single-photon emission computed tomography (SPECT) in nuclear cardiology. During this period, there was a shift from Anger-type gamma cameras equipped with one detector to those with multiple detectors. Semiconductor detectors have also become available in recent years. Devices with $\mathrm{X}$-ray CT and positron emission tomography (PET) -compatible ones are also now available. There was also a shift in image processing: from the filtered back projection (FBP) to the ordered-subsets expectation maximization (OS-EM) method. Since the OS-EM method improves the quality of images as one of its characteristics, the frequency of sampling and collection time is being reviewed to determine the values appropriate for the reconstruction method. Types of OS-EM method that have incorporated attenuation, scatter, and resolution corrections have also been in practical use. This technological progress has improved the quality of SPECT images and diagnostic accuracy, and the use of nuclear cardiology SPECT will be further promoted.

Keywords: Myocardial SPECT, SPECT system, Acquisition condition

Ann Nucl Cardiol 2015 ; 1 (1) : 132-135

I

n Japan, single-photon emission computed tomogra-

phy (SPECT) using a gamma camera has been reported on since 1975 (1). Initially, the gamma camera was fixed and the patient sat on a revolving chair that was turned manually. The amount of rotation was set by referring to a protractor scale. Later, the method changed; the patient was stationary and the operator now turned the gamma camera. The data acquisition method of nuclear cardiology testing has also changed, based on the number of gamma camera detectors.

Although there are many manuals explaining the changes in the structure of SPECT systems, not many manuals refer to the conditions of data acquisition. This paper describes appropriate conditions of data acquisition for two different reconstruction methods: the filtered back projection $(\mathrm{FBP})$ reconstruction and the ordered-subsets expectation maximization (OS-EM) iterative reconstruction.

\section{SPECT system}

With one detector, data are acquired during 180-degree rotation from right anterior oblique (RAO) 45 degrees to left posterior oblique (LPO) 45 degrees. With two detectors, data are acquired over 360 degrees and the detectors are set opposite each other. With three detectors, data are acquired over 360 degrees and the detectors are arranged to form a triangle. The body thickness of an average Japanese person is less than that of an average European or American. Therefore, the heart can be well imaged by a posterior view. Furthermore, incomplete sampling of the projection data in 180-degree acquisition degrades image quality.

\footnotetext{
doi : 10.17996/ANC. 01.01.132

Yasuyuki Takahashi

Department of Nuclear Medicine Technology, Gunma Prefectural

College of Health Sciences, 323-1 Kamioki-cho, Maebashi,

Gunma, Japan 371-0052

E-mail: takahashi-yasuyuki2@gchs.ac.jp
} 
Therefore, 360-degree acquisition has become popular in Japan. However, since the European congress of radiology (ECR) guidelines of 2003 (2) recommended 180-degree acquisition, this type of acquisition is still employed when using two detectors in Japan, by setting the detectors in a L-shape, or at a camera-specific angle. Currently, for SPECT systems with semiconductor detectors, 180-degree acquisition is being used.

A dedicated semiconductor SPECT system has also been developed for cardiac SPECT (3). The detector is L-shaped and does not rotate during the scan. This system has four characteristics that differ from conventional Anger-type camera systems. First, the sensitivity is improved by a factor of more than four, allowing the SPECT scan time to be reduced. Second, spatial resolution is improved by a factor of more than two, so diagnostic accuracy is improved. Third, energy resolution is improved, so a simultaneous dual isotope scan employing ${ }^{99 \mathrm{~m}} \mathrm{Tc}$ and ${ }^{123} \mathrm{I}$ becomes possible. Fourth, the Lister function improves time resolution; this is an important function to quantify myocardial perfusion. In addition, the semiconductor detector easily accommodates the prone position. In this system, images are reconstructed by use of a 3-D iterative Baysian reconstruction algorithm incorporating 19 projection data obtained by 19 fixed detectors mounting pinhole collimators. The collimation is focused on the myocardium. Semiconductor SPECT is different from conventional one in projection data.

In terms of other systems, for positron emission tomography (PET)/SPECT systems, two acquisition methods can be chosen. One is PET alone coincidence acquisition using Septa. The other is equipped with an ultra-high-energy high-resolution collimator and involves dual-radionuclide simultaneous acquisition of SPECT and PET. Simultaneous acquisition is recommended because two kinds of image without misregistration are provided in dual-radionuclide simultaneous acquisition.

For all SPECT systems, the way of thinking about the acquisition conditions is the same.

\section{Acquisition conditions}

Pixel size influences the reproducibility of the signal. The pixel size should meet the criterion of the FWHM being $\leqq 2.5$ pixels. When the myocardial thickness is approximately $10 \mathrm{~mm}$, a pixel size of $3.2-6.4 \mathrm{~mm}$ is used.

As for the matrix size, with 3 detectors, it is $128 \times 128$, and with 2 detectors, it is $64 \times 64$ and amplification is added. The amplification makes the pixel size more appropriate; a value between 1.3 and 1.5 is usually used. The value is chosen to allow the heart projection to fall within the effective field of view of the gamma camera.

The sampling angle and the count number influence image quality. The required sampling angle can be calculated from sampling theorem. Sampling theorem requires $\mathrm{N} \geqq \pi \mathrm{D} / 2 \mathrm{a}$. (Here, $\mathrm{N}$ is the number of samples, $\pi \mathrm{D}$ is the length of the circumference, and $\mathrm{a}$ is the pixel size.)

However, this theorem is adapted when the count number is sufficient. Furthermore, image quality is also affected by a pre-filter. Actually, owing to the need to acquire SPECT data within a reasonable amount of time, generally, the sampling angle step size is between 4 and 6 degrees (4).

\section{Image processing}

The acquisition conditions have been discussed for the FBP method. However, the acquisition conditions can be reviewed for maximum likelihood expectation maximization (ML-EM) and OS-EM reconstruction (5). Fig. 1 shows the myocardial SPECT image produced when using a number of samplings of 30,60 , or 90 projections. When there are 30 projections, an aliasing artifact occurs for FBP reconstruction. However, the SPECT images are improved by OS-EM reconstruction. Fig. 2 shows the myocardial SPECT image produced by 60 angular samples, but with a mean myocardial count of 10 , 50, or 100 counts/pixel per projection. When the count density is low, an aliasing artifact again occurs. However, the SPECT images are improved by OS-EM reconstruction, as was the situation with 30 projections (Fig. 1).

Scatter-, attenuation- and spatial resolution correction are required for myocardial SPECT in order to improve the accuracy of quantification. The dual-energy window subtraction (DEWS) method (6), the effective scatter source estimation (ESSE) method (7), and the triple-energy window (TEW) method (8) are used in scatter correction. The Chang method (9), the Segmentation with Scatter and Photopeak window data for Attenuation Correction (SSPAC) method (10), and the X-ray CT method (11) are used in attenuation correction. The Frequency-Distance Relationship (FDR) method (12) and the collimator board correction (CBC) (13) method are used in spatial resolution correction. These correction methods have recently been incorporated in an iterative algorithm (14). In addition, it has recently been argued that a correction for the partial volume effect (15) is also necessary. See Fig. 3 to observe the effect of each type 
FBP

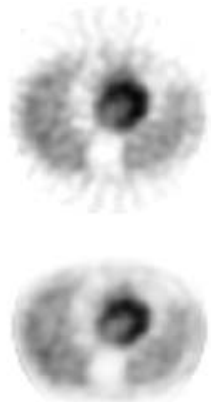

30
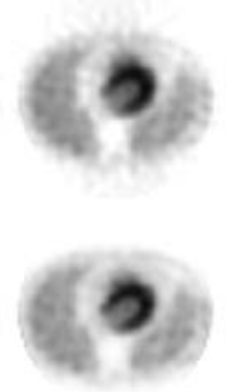

60
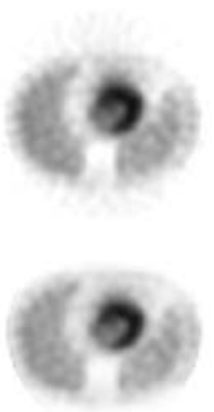

120

number of projections
Fig. 1 Different SPECT images produced by different numbers of angular samples. The image reconstruction method is a parameter. Top row, FBP. Bottom row, OS-EM.

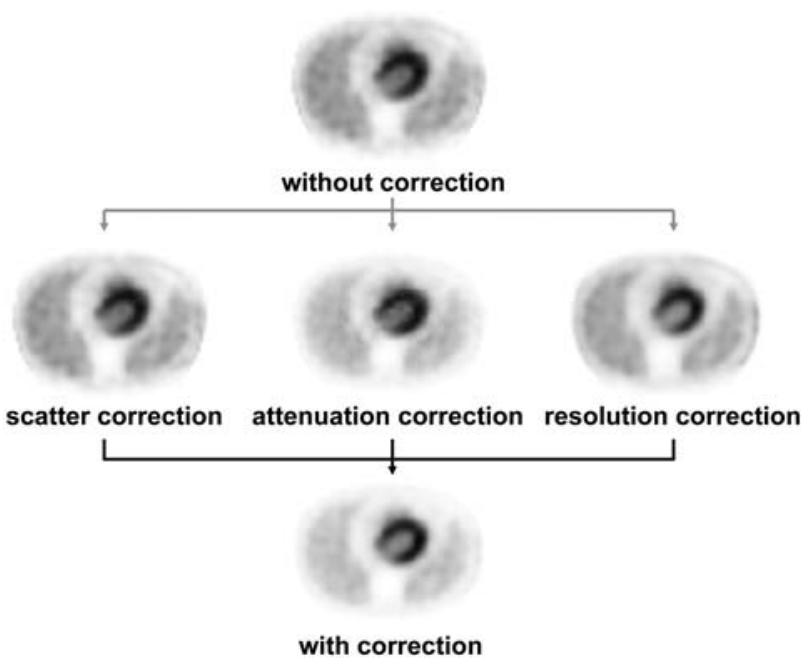

Fig. 3 Different SPECT images produced by different corrections, from none to all, as labeled.

of correction.

Although the present system with semiconductor detectors compensates photon scattering by using the DEWS method, attenuation correction is not available. Instead another version equipped with CT was released, which allows the attenuation map for correction.

\section{Image display}

Finally, this section deals with the issue of image display. The scale employed relates the image brightness to the numerical value reconstructed for the voxel. In myocardial SPECT, the typical scale uses a linear or quadratic relationship. Contrast is cut about $10 \%$ to $20 \%$ in many cases. However, a linear scale that is not cut can also display the background data exactly. As quantitative gated SPECT (QGS) (16), in addition to conventional vertical long axis (VLA), short axis (SA), and horizontal long axis (HLA), the Bull's eye map is common. SPECT image is displayed with division into
FBP
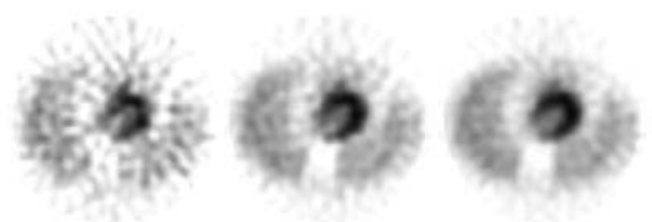

OSEM

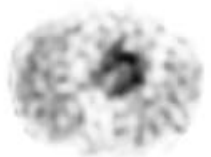

10

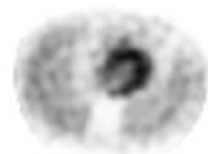

50

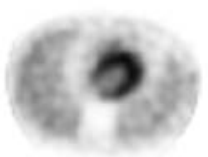

100

pixel counts / projection
Fig. 2 Different SPECT images produced by different pixel count densities. The image reconstruction method is a parameter. Top row, FBP. Bottom row, OS-EM.

9-16 with 1 slice of 5-7 mm. With the ${ }^{99 \mathrm{~m}} \mathrm{Tc}-$ radionuclide, the influence of high accumulation in the liver must be reduced. Therefore, the frequency of projection of the myocardium was regarded as the maximum count when displaying images. If the frequency of projection was higher for the liver than for the myocardium when restructuring images using projection data, the counts for the liver and myocardium were regarded to be similar.

\section{Conclusions}

It is desirable for myocardial SPECT images to be acquired in the shortest time while still yielding sufficient image quality to allow high diagnostic accuracy. Therefore, there is a need to choose appropriate acquisition conditions in order to provide good reproducibility and accuracy.

\section{Acknowledgment}

The authors thank Teruhito Mochizuki (Ehime University Graduate School of Medicine), Keigo Endo (Kyoto College of Medical Science), the study participants, and Toshiba Medical Systems Corporation and GE Healthcare for their support during these studies.

I received the 3rd Japanese Society of Nuclear Cardiology Technology Award for my research on the improvement of SPECT image quality.

\section{Sources of Funding}

This study was supported by JSPS Grants-in-Aid for Scientific Research (C) in Japan (grant number 24500823).

\section{Conflicts of Interest}

None 
Reprint requsts and correspondence :

Yasuyuki Takahashi,

Department of Nuclear Medicine Technology, Gunma

Prefectural College of Health Sciences, 323-1

Kamioki-cho, Maebashi, Gunma, Japan 371-0052

E-mail : takahashi-yasuyuki2@gchs.ac.jp

\section{References}

1. Akiyama Y, Kinoshita F, Koakutsu M, et al. The stories of first machines in Japan -SPECT-. Jpn J Radiol Technol 2001; 57: 372-6. (in Japanese)

2. Hesse B, Tagil K, Cuocolo A, et al. EANM/ESC procedural guidelines for myocardial perfusion imaging in nuclear cardiology. Eur J Nucl Med Mol Imaging 2005; 32: 855-97.

3. Takahashi Y, Miyagawa M, Nishiyama Y, et al. Performance of a semiconductor SPECT system: comparison with conventional Anger-type SPECT. Ann Nucl Med 2014; 27: 11-6.

4. Takahashi Y, Murase K, Mochizuki T, et al. Evaluation of the number of SPECT projections in the ordered subsets-expectation maximization image reconstruction method. Ann Nucl Med 2003; 17: 525-30.

5. Hudson HM and Larkin RS. Accelerated image reconstruction using ordered subsets of projection data. IEEE Trans Med Imaging 1994; MI-13: 601-9.

6. Jaszczak RJ, Greer KL, Floyd CEJr, et al. Improved SPECT quantification using compensation for scattered photons. J Nucl Med 1984; 25: 893-900.

7. Frey EC, Tsui BMW. A new method for modeling the spatially-variant, object-dependent scatter response, function in SPECT. IEEE Nucl Sci Symp 1996; 2: 1082-6.

8. Ogawa K. Simulation study of triple-energy-window scatter correction in combined Tl-201, Tc-99m SPECT. Ann Nucl Med 1994; 8: 277-81.

9. Chang LT. A method for attenuation correction in radionuclide computed tmography, IEEE Trans Nucl Sci 1978; NS-25, 638-43.

10. Yamauchi Y, Kanzaki Y, Otsuka K, et al. Novel attenuation correction of SPECT images using scatter photopeak window data for the detection of coronary disease. J Nucl Cardiol 2014; 21: 109-17.

11. Patton JA, Delbeke D, Sandler MP. Image fusion using an integrated, dual-Head coincidence camera with $\mathrm{X}^{-}$ ray tube-based attenuation maps. J Nucl Med 2000; 41: 1364-8.

12. Edholm PR, Lewitt RM and Lindholm B. Novel properties of the Fourier decomposition of the sonogram. Proc. SPIE1986; 671: 8-18.

13. Takahashi Y, Murase K, Mochizuki T, et al. Simultaneous three-dimensional resolution correction in SPECT reconstruction using OS-EM algorithm. J Nucl Med Tech 2007; 35: 34-8.

14. El Fakhri G, Buvat I, Benali H, et al. Relative impact of scatter, collimator response, attenuation, and finite spatial resolution corrections in cardiac SPECT. J Nucl Med 2000; 41: 1400-8.

15. Hutton BF, Osiecki A. Correction of partial volume effects in myocardial SPECT. J Nucl Cardiol 1998 5: 402-13.

16. Germano G, Kavanagh PB, Su HT, et al. Automatic reorientation of three-dimensional, transaxial myocardial perfusion SPECT images. J Nucl Med 1995; 36: 1107-14. 